\title{
Análisis del modelo de gestión de la comunicación y las RRPP de las ONG en Valencia en una sociedad en crisis (2012)
}

\section{Valentzian, krisi betean dagoen gizarte batean, GKEk harreman publikoak eta komunikazioa kudeatzeko erabilitako ereduaren azterketa (2012)}

\section{Analysis of the management model of communication and PR of NGO in Valencia in a society in crisis (2012)}

\section{Elisa Regadera González ${ }^{1}$ M. Pilar Paricio Esteban² Hildegart González Luis ${ }^{3}$}

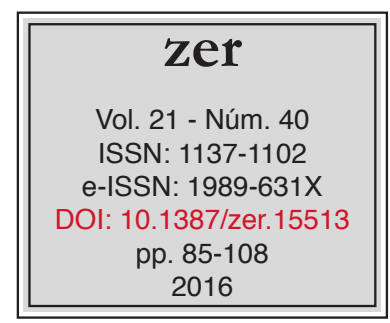

Recibido el 28 de octubre de 2014, aceptado el 18 de noviembre de 2015.

\section{Resumen}

Este artículo se orienta a conocer la gestión de la comunicación realizada por las organizaciones del Tercer Sector durante la crisis económica, que ha puesto en valor el trabajo de estas entidades en un momento de déficit de confianza y pérdida de credibilidad en las instituciones. El trabajo trata de analizar el modelo de gabinete y la gestión de la comunicación desarrollada por seis ONG de diferente perfil que trabajan con colectivos en riesgo de exclusión social en Valencia (Cruz Roja, Cáritas, Casa Caridad, CEARPV, COCEMFECV y FEAPSCV), abordando la situación en una comunidad especialmente castigada por la crisis.

Palabras clave: Gabinete de comunicación, ONG, crisis económica, plan de comunicación, relaciones públicas.

\footnotetext{
Universidad Católica de Valencia, elisa.regadera@ucv.es

2 Universidad CEU Cardenal Herrera, pparicio@uch.ceu.es

3 Universidad de Navarra, hgonzal@ unav.es
} 


\title{
Laburpena
}

Artikulu honen helburua da gertuagotik aztertzea nola kudeatu duten komunikazioa Hirugarren Sektoreko erakundeek krisialdi ekonomikoak iraun bitartean. Azterlanak balioa eman dio erakunde horien lanari, herritarrek konfiantzarik ez zuten eta erakundeek sinesgarritasuna galdu duten garai batean. Egindako lanaren helburua da Valentzian gizartetik at gelditzeko arriskuan dauden kolektiboekin lan egiten duten hainbat profiletako sei GKEk (Gurutze Gorria, Cáritas, Casa Caridad, CEARPV, COCEMFECV eta FEAPSCV) gauzatu duten komunikazioaren kudeaketa eta kabinetearen eredua aztertzea, kontuan izanik krisiak bereziki gogor jo duela Valentzia.

Gako-hitzak: Komunikazio kabinetea, GKE, krisi ekonomikoa, komunikazio plana, harreman publikoak.

\begin{abstract}
This paper aims to know the communication management by Third Sector organizations during the economic crisis, which has value to the work of these entities in a moment of trust deficit and loss of credibility in the institutions. The paper analyzes the model of staff and management of communication developed by six different profile NGOs working with groups at risk of social exclusion (Red Cross, Caritas, Charity House, CEARPV, COCEMFECV and FEAPSCV) in Valencia, tackling the situation in an autonomous region particularly affected by the crisis.
\end{abstract}

Keywords: Communication departments, NGOs, economic crisis, communication plan, public relations. 


\section{Introducción}

La crisis económica que atraviesa España desde 2008, cuyas consecuencias más duras se han sufrido en 2012 y 2013, ha enfrentado a las ONG a dos movimientos de signo contrario: por un lado, un incremento de la demanda de sus servicios motivada por el aumento de las necesidades sociales; y, por otro, una disminución de sus recursos financieros debido a las fuertes restricciones presupuestarias de las Administraciones Públicas, que repercute negativamente en sus ingresos ${ }^{4}$.

Unido a ello, el protagonismo de una sociedad civil no estructurada, la crisis de confianza y credibilidad a la que están sometidas las ONG, y la demanda de transparencia y responsabilidad que se les exige, acentúan la necesidad de garantizar una comunicación eficaz y coherente con su quehacer institucional, que no puede limitarse a una mera transmisión informativa o a estratagemas persuasivas que desequilibran la relación con los públicos, pues éstos demandan ser interlocutores activos y solicitan poder contribuir y participar en la toma de decisiones (Gutiérrez-García y Laporte, 2013).

En este sentido, los nuevos retos planteados a las ONGs para asegurar la supervivencia en una sociedad en crisis pasan por entender la comunicación no solo como función técnica, sino como función directiva y estratégica (Sriramesh y Vercic, 2012), entendida como capacidad proactiva para acercar a los públicos a las decisiones estratégicas propiciando su colaboración (Canel, 2007).

Se evidencia así la importancia de la gestión de la comunicación y relaciones públicas en estas organizaciones, fundamentada en "una planificación de mensajes clave dirigidos a públicos estratégicos que permitan comprometer a sus integrantes" (Durán y Fernández, 2010: 603). Es decir, una comunicación dirigida a sensibilizar e implicar a la sociedad en el apoyo a los colectivos más vulnerables ante la crisis, además de captar socios y recursos humanos (voluntarios) y económicos que les permitan mantenerse y desarrollar su trabajo.

\section{La comunicación en las ONG españolas}

\subsection{Los departamentos de comunicación y relaciones públicas}

A la hora de abordar la gestión de la comunicación en las organizaciones del Tercer Sector, debemos partir del análisis del origen y funcionamiento de los departamentos de comunicación y relaciones públicas en estas entidades.

Existe una amplia bibliografía sobre las relaciones públicas y los gabinetes de comunicación, tanto a nivel internacional como en España. Algunos de los estudios más relevantes en nuestro país profundizan en el concepto de gabinete de comunicación, subrayando su función como gestores de información y su evolución hacia el concepto gerencial (Ramírez, 1995: 27; Martín, 2006; Castillo, 2009), su orientación hacia la planificación estratégica (Pérez Varela, 1996: 149; García, 2005: 74), el rol de la dirección de la comunicación en las organizaciones (Villafañe, 2008), y sus implicaciones con la imagen y reputación corporativas (Costa, 2009; L'Etang, 2009).

\footnotetext{
${ }^{4}$ Fundación Luis Vives, Revista Española del Tercer Sector, $n^{\circ}$ 23. Informe sobre el impacto de la depresión económica en el Tercer Sector de Acción Social (2013).
} 
Algunos autores plantean diferentes tipologías de gabinetes de comunicación y analizan sus particularidades. En función de la ubicación del departamento, diferencian entre gabinetes internos y externos (Wilcox, 2012); en función de la organización, entre: gabinetes de administraciones públicas o instituciones (Xifra, 2008; Almansa, 2011; Wilcox, Cameron y Xifra, 2012), gabinetes en empresas (Martín, 2006; Pagán, 2009) y gabinetes de organizaciones sociales (Bel, 2004; García, 2005; González, 2006; Balas, 2011).

Diversas investigaciones profundizan, además, en el origen y evolución de los departamentos de comunicación y las diferentes funciones que asumen (Cervera, 2008). Los primeros departamentos de comunicación surgen como gabinetes de prensa, observándose una evolución del gabinete de prensa al de comunicación (Almansa, 2011; Balas, 2011).

Si nos aproximamos al estudio de los gabinetes de comunicación en organizaciones del Tercer Sector, en los últimos años encontramos varias tesis doctorales que se refieren a la comunicación en estas entidades (Fernández, 2004; González, 2005; Balas, 2010; Herranz de la Casa, 2010; González, 2010; Regadera, 2014). También hay que destacar las publicaciones que abordan el papel determinante de los Dircom en los gabinetes de comunicación de las ONG y los rasgos que definen sus perfiles (Molina, 2007: 291; Álvarez, 2012: 247; Regadera y Paricio, 2014; Paricio y Regadera, 2014). Por último, merece la pena señalar que existen diversos informes de interés a nivel nacional o en otras Comunidades autónomas que también tratan la situación comunicativa de estas entidades: CONGDE ${ }^{5}$ y Fundación Hazloposible Bidea ${ }^{6}$. Y en la Comunidad Valenciana, Fundar ${ }^{7}$. Las investigaciones realizadas hasta el momento constatan que si el trabajo que desarrollan las ONG ha penetrado durante las últimas décadas en el tejido y la conciencia social ciudadana ha sido, en gran parte, gracias a la tarea desarrollada desde los gabinetes de comunicación. Además, confirman que los Gabinetes de las ONG son de más tardía creación que en otros sectores (García, 2005), y que para ser realmente eficaces deben procurar adaptarse continuamente a un entorno cambiante (Bel, 2004).

El origen de los gabinetes de comunicación de las ONG españolas se sitúa en los años 90 (García, 2006; González, 2006), por lo que podemos señalar que se trata de departamentos jóvenes y con una trayectoria corta, que podría influir en muchos casos en el nivel de profesionalización y/o recursos de los mismos. Según concluyen diversos estudios, es en esa década cuando las ONG más desarrolladas comienzan a darse cuenta de que sólo siendo transparentes y comunicando de forma clara y estratégica, conseguirán los apoyos necesarios para crear una sólida base social y consistencia económica. En 1997 el 90\% de las ONG registradas en las CONGDE contaban con un departamento de comunicación. Según García (2006), los principales motivos que impulsaron la creación de dichos gabinetes fueron la necesidad de

\footnotetext{
5 CONGDE (Coordinadora de ONG para el Desarrollo-España). Informe sobre los medios de comunicación y las ONGD. Situación actual y retos (2007). Recuperado de: http://www.congde.org/ contenidos/descargar/attachedfiles/962/original?1363750391

${ }^{6}$ Fundación Hazloposible y Comunidad de Madrid. Informe sobre las necesidades comunicativas de las ONG (2011). Consultora Bidea.

7 FUNDAR. Informe-Evaluación sobre la capacidad comunicativa del sector asociativo en la Comunidad Valenciana (2011). Recuperado de: http://www.fundar.es/descargas/varios/estudio_comunicacion_definitivo.pdf
} 
ser más conocidas por parte del público, la toma de conciencia sobre la importancia de la comunicación en el desarrollo de su misión, y la necesidad de agrupar bajo una misma estructura su diversidad de funciones.

La revisión bibliográfica y de las investigaciones previas confirma también que estos departamentos en las ONG surgen en algunos casos como gabinetes de prensa (Almansa, 2011). Algunos expertos, como Balas (2011), opinan que en el siglo XXI sus responsables se dedican mayoritariamente a la gestión de las relaciones con los medios -atendiendo a su perfil periodístico, y a las tácticas y públicos a los que se dirigen-, lo que les aproxima más a un modelo de gabinete de prensa. Originariamente se utilizaba el término "gabinete de prensa" para designar todas las funciones de comunicación interna y externa de un departamento de comunicación, pero en realidad un gabinete de prensa es fundamentalmente la oficina que conecta la información de una organización con la opinión pública a través de los medios de comunicación, y su estructura suele ser: Jefe de prensa, periodistas y ayudantes (Aragonés, 1998).

Sin embargo, en la última década Almansa (2011) apunta la evolución del gabinete de prensa al gabinete de comunicación en las organizaciones, con perfiles profesionales más completos, una función estratégica y no meramente técnica, y una orientación a la generación de relaciones con todos los stakeholders de la organización. Bell (2004) coincide también destacando dos áreas principales en la gestión de los gabinetes: comunicación interna y externa, asumiendo funciones más allá de las relaciones con los medios, que se integrarían en la gestión de las relaciones externas. Otros autores inciden también en que podrían tener una estructura de áreas y funciones similares a las de otras organizaciones públicas y privadas, como las propuestas por Ramírez (1995), Cervera (2008), Almansa (2011) y Balas (2011): comunicación interna; comunicación externa; marketing y publicidad; relaciones con la sociedad/ comunidad y comunicación de crisis.

La comunicación interna es un ámbito práctico y de investigación en auge, dada la notoria importancia que este público adquiere para el éxito de empresas y entidades en las dos últimas décadas (Del Pozo, 2000; Villafañe, 2008; Pagán, 2009). En lo que respecta a las $\mathrm{ONG}$, en la bibliografía revisada se detecta que tienen públicos internos con peculiaridades distintas a las de otras entidades - personal no remunerado, como los voluntarios, o contrapartes que ejecutan su labor a miles de kilómetros-.

En lo referente a la comunicación externa, los investigadores coinciden en señalar que las ONG deben potenciar su estrategia de actuación ante la sociedad, ante las instituciones, y sobre todo ante los medios de comunicación, para satisfacer determinadas finalidades como la captación de socios, colaboradores y voluntarios, la sensibilización social, o las relaciones institucionales (Romero, 2008).

Con respecto al marketing y la publicidad, los departamentos de finanzas y marketing de las ONG han de estar íntimamente relacionados con el de comunicación. En este sentido, Bernabé (2001) destaca que el objetivo de la mayoría de las ONG con un área de comunicación es conseguir financiación para potenciar su independencia, por lo que a veces se priman líneas publicitarias y de marketing por encima otros ámbitos comunicativos e informativos. En lo que respecta al área de relaciones con la sociedad/comunidad, son varios los autores que incluyen estas acciones en el ámbito de la comunicación externa. Por ejemplo la organización de eventos 
está relacionada frecuentemente con la captación de fondos y/o la información/ fidelización de los socios, donantes, voluntarios, etc.

Por último, la comunicación de crisis en las ONG, más que un área diferenciada, podría ser considerada una función de la comunicación que es preciso determinar. Las entidades no lucrativas han de mejorar la planificación de los pasos a seguir y las medidas comunicativas que deberán poner en funcionamiento ante estas eventuales situaciones.

\subsection{Crisis económica y planificación estratégica de la comunicación}

Desde 2007 España está inmersa en una crisis económica que ha afectado a porcentajes cada vez mayores de la población. En 2012, el 21,1\% de los españoles se encontraba en situación de pobreza (Sánchez Morales, 2013: 51), y según el Instituto Nacional de Estadística (INE 2013: 28), España tenía ese año un 25\% de desempleo, la cifra más elevada de la Unión Europea. Son varios los autores que apuntan que el paro de larga duración, junto al endeudamiento en la adquisición de viviendas y la reducción del gasto social (40\% menos en 2013 para servicios sociales básicos), han sido los principales desencadenantes del tránsito hacia la pobreza y la exclusión social que ha afectado a muchas personas y familias (Gutiérrez-Domenech, 2013).

Esta situación ha dado lugar a la aparición de los "nuevos pobres", personas sin antecedentes de exclusión ni desadaptación que, como consecuencia de la crisis, se han visto abocados -según el Observatorio de la Realidad Social de Cáritas Española- primero a buscar apoyo en sus familias, seguido del trabajo en la economía sumergida, y en último lugar a la búsqueda de apoyo por parte de organizaciones sociales (Caritas 2011: 12).

Una de las comunidades más afectadas por la crisis ha sido la valenciana. Así, según señala el VII Informe FOESSA sobre exclusión y desarrollo social en España $(2014)^{8}$, si comparamos la situación de integración social a escala estatal respecto a la situación encontrada a escala autonómica en 2013, los hogares situados en Valencia se encuentran globalmente en una situación de mayor vulnerabilidad y exclusión (el 64,9\% no están plenamente integrados frente al 63,1\% en España). Por tanto, el $14,5 \%$ de los hogares en Valencia se sitúan en la exclusión moderada (1,4 puntos más por encima del total estatal) y el $11,5 \%$ en la exclusión severa (2,7 puntos porcentuales más).

La crisis económica ha sido una oportunidad para el reconocimiento social de la labor de las ONG, a la par que se ha incrementado sustancialmente su labor con personas vulnerables y en riesgo de exclusión, mientras la Administración ha reducido sustancialmente las ayudas a estas entidades (Informe Luis Vives, 2013). Ante esta situación, las ONGs necesitan articular una política de comunicación que les ayude a mostrar el impacto social y económico de su labor, generar notoriedad y diferenciación, así como confianza en sus públicos y una reputación positiva.

Como se ha expuesto anteriormente, los gabinetes de comunicación son una realidad extendida también en el sector de las ONG, pero diversos estudios evidencian que uno de sus retos a afrontar desde comienzos del siglo XXI es la

\footnotetext{
8 Informe sobre exclusión y desarrollo social en la Comunidad Valenciana (2014). Avance de resultados de la Encuesta sobre Integración y Necesidades sociales, 2013.
} 
comunicación planificada desde un enfoque estratégico con todos sus públicos (De la Riva, 2002).

Existe una amplia variedad de investigaciones elaboradas desde diversos ámbitos sobre qué es y cómo se elabora una estrategia de comunicación (Bernays, 1996; Matilla, 2007; Pérez, 2008). En este estudio seguiremos la propuesta por Matilla (2007), quien señala que si bien la comunicación ha sido considerada durante mucho tiempo como transmisión de conocimientos, tras las recientes transformaciones sociales, económicas y organizacionales producidas en las últimas décadas, ha adquirido una dimensión más amplia que implicará intercambios informativos con el objetivo específico de modificar los comportamientos de los receptores; es decir, se enmarcará en un plano persuasivo de relaciones interactivas presididas por un planteamiento estratégico. La estrategia se concentra -según esta autora- en la forma de eludir o sortear una situación amenazante real en un contexto coyuntural determinado, que será dilucidado mediante un plan de acción.

Desde esta perspectiva, esta investigación considera que los gabinetes de comunicación en las ONG se demuestran especialmente necesarios para conseguir diferentes objetivos con sus públicos de interés. Gestionar la comunicación en las ONG consistirá por tanto en planificar "mensajes clave dirigidos a públicos estratégicos que permitan comprometer a sus integrantes, logrando la productividad y sensibilizando a la opinión pública" (Durán y Fernández, 2010: 603).

Sin embargo, a pesar de que desde la década de los 90 los recursos destinados a la comunicación aumentaron progresivamente, autores como Erro y Ventura (2002: 107) señalan que el flujo comunicativo en muchas ONG españolas todavía no circula suficientemente por "la falta de planificación y coordinación en la comunicación interna, tanto entre las diferentes áreas como entre los miembros (socios, voluntariado, etc.), la escasa comunicación con las contrapartes, o la casi inexistente relación con los movimientos sociales".

Otros estudios, sin embargo, (Balas, 2011; Herranz de la Casa, 2009; González, 2006) detectan una mejoría en este campo y apuntan cómo los gabinetes de comunicación entran a formar parte progresivamente de la estrategia de las ONG, tanto para mejorar su reputación, como encaminados al logro de sus fines y objetivos, hasta llegar a convertirse incluso en elementos de presión: "Con frecuencia, dos o tres años después de la creación y consolidación del departamento de comunicación, cada institución incorpora la primera estrategia comunicativa" (González, 2006: 79). Además, consideran que la comunicación en las ONG se está convirtiendo cada vez más en una herramienta estratégica, no sólo porque contribuye a formar la imagen deseada de estas organizaciones en los mercados (Cornelissen y Harris, 2001), sino también porque les permite influir sobre ellos a través de una comunicación bidireccional con sus diferentes públicos, como parte esencial de sus valores como entidades que prestan servicios a la sociedad.

Balas (2011: 201) cita a Vernis et al. (1998) -que elevaba la función de comunicación en las ONG a un terreno estratégico- al definir el sentido de la comunicación de las entidades no lucrativas como su razón de ser: “¿habéis pensado alguna vez que las Asociaciones existen para comunicar, mientras que las empresas comunican para existir y para conseguir un beneficio? Dicho de otra manera, las organizaciones no lucrativas existen para transmitir sus valores centrales a la sociedad". 
El diseño estratégico de la comunicación y de las relaciones públicas en cualquier entidad suele plasmarse normalmente en el Plan de comunicación, como herramienta utilizada para la planificación, desarrollo y ejecución de cualquier estrategia comunicativa, sobre el que diversos autores han investigado (Bel, 2004; Martin, 2006; Cervera, 2008; Matilla 2009; Wilcox et al., 2012). Su configuración ha sido delimitada por diversos autores, dando lugar a múltiples modelos de planificación.

En esta investigación, adoptaremos el modelo RACE propuesto en 1963 por John Marston en su libro The Nature of Public Relations. En él, fundamentándose en el proceso de management por objetivos -como recopilación de información para ser analizada, aplicando un plan de acción vinculado a los mismo- (Matilla, 2011), estableció el proceso de toma de decisiones estratégicas en el ámbito de las relaciones públicas y lo estructuró en cuatro etapas, a las cuales denominó con el acrónimo "RACE": Research (Investigación), Action (Acción), Communication (Comunicación), Evaluation (Evaluación). Este método -también llamado "Método de la espiral"- es uno de los más utilizados en el desarrollo de la gestión profesional de la función de la comunicación en las organizaciones y uno de los más citados en el ámbito académico e inspirador de la mayoría de las propuestas de planificación estratégica desarrolladas por diversos autores (Cervera; 2008; Fernández del Moral, 2005; Xifra, 2008).

Matilla define la investigación, como "la búsqueda controlada de información objetiva y sistemática" (2011: 84) jugando un papel importante el análisis DAFO, que ayuda a determinar las estrategias y medios más oportunos en la fase de planificación o acción. Comprender a los públicos, evitar errores, tomar decisiones ajustadas y conectar con el entorno, son algunas de las justificaciones, según Xifra (2005), del porqué es esencial investigar antes de diseñar el nuevo plan de comunicación y relaciones públicas. Esta fase -según Cultip y Center (1952)- consiste en relacionar las actitudes de los públicos descubiertas en la fase de investigación, con los programas de la organización fijando los objetivos de comunicación. La segunda fase del proceso de planificación estratégica recibe el nombre de acción: "es por medio de la acción como el sujeto establece un diálogo permanente con su entorno" (Castillo y Alvárez, 2015). Aquí la delimitación de objetivos permitirá desarrollar los dos procesos comunicativos claves: política de comunicación y plan estratégico de comunicación. La etapa de comunicación responde a la fase de elaboración de la estrategia propiamente dicha (Castillo y Alvárez, 2015), donde se eligen los medios y acciones estableciendo mecanismos para comunicar interna y externamente el mensaje (Xifra, 2005). Además -apunta Matilla (2007)- incluye la confección del calendario del plan de acción (táctico).

La última etapa es la de evaluación, dond, siguiendo a Pavlik (1999), conviene diferenciar entre evaluación formativa -que describe si un programa o plan posee unos objetivos concretos y mensurables antes de ser implementado- y formativa -que sirve para determinar la forma en que se alcanzan los objetivos-. En los diferentes modelos de planificación estratégica desde la perspectiva RACE (Hainsworth y Wilson, 1992; Black, 1994), la evaluación se orienta a examinar los resultados obtenidos, pero, como apunta Krohling Kunsch (2005), forma parte de todo el proceso. Además, existen otros modelos que inciden en diferentes niveles de evaluación, como Lindenmann en su modelo "Public Relation Effectiveness Yarstick", donde 
habla de nivel básico (se mide producción), intermedio (midiendo si los mensajes han sido recibidos, comprendidos y retenidos) y avanzado (mide resultados en cambios de opinión y comportamientos.

\section{Metodología}

El presente artículo se orienta a conocer la gestión de la comunicación realizada por las organizaciones del Tercer Sector, en una situación de profunda crisis económica que ha puesto en valor el trabajo de estas entidades, dando respuesta a necesidades sociales no cubiertas desde los estamentos públicos en un momento de déficit de confianza y pérdida de credibilidad en las instituciones. Esta situación, junto con los retos de una sociedad globalizada y altamente competitiva donde crece el protagonismo de la sociedad civil y la exigencia de organizaciones responsables y transparentes, acentúa la necesidad de una comunicación eficaz. Partiendo de estas premisas y de los antecedentes expuestos en el marco teórico, el objeto de este trabajo es el análisis de la gestión de la comunicación desarrollada por las ONG durante la crisis económica que atraviesa España, partiendo de algunas ONG que trabajan de forma representativa con colectivos en riesgo de exclusión social, en una comunidad autónoma especialmente castigada por la crisis como es la Comunidad Valenciana.

Si tenemos en cuenta las escasas investigaciones desarrolladas en España sobre el tema objeto de estudio durante la crisis y la falta de estudios al respecto en dicha comunidad, queda justificada la oportunidad de esta investigación, cuyos resultados mejorarán el conocimiento de la gestión de la comunicación realizada por las entidades objeto de estudio, ofreciendo datos actuales sobre su trayectoria, estructura y funciones, procesos de trabajo y modelos seguidos en la planificación estratégica de su comunicación.

Como hipótesis de partida se presupone que el modelo de gestión de la comunicación en las ONGs ha evolucionado del gabinete de prensa a un gabinete de comunicación integral, con un planteamiento estratégico de la comunicación en línea con el método RACE. Esta hipótesis se sustenta en las siguientes premisas:

- La gestión de la comunicación en las entidades del Tercer Sector ha experimentado en los últimos años una creciente profesionalización, dando lugar a departamentos internos o externos.

- La escasez de recursos de las ONG, especialmente en momentos de crisis económica, puede favorecer que la comunicación sea considerada una herramienta clave al servicio de los fines de estas entidades, pasando de un planteamiento táctico a otro estratégico.

- El modelo RACE es uno de los más citados en el ámbito académico, sirviendo de base a otros modelos (Xifra, 2008; Matilla, 2009) y siendo uno de los más utilizados en la gestión profesional de la comunicación en diferentes tipos de organizaciones. 
Teniendo en cuenta la hipótesis de partida, el objeto de la investigación es conocer el modelo o modelos de gestión de la comunicación predominantes en las ONG en una situación de crisis económica, realizando un pilotaje centrado en la Comunidad Valenciana y planteando una metodología que pueda ser replicada en estudios posteriores en otras comunidades.

Para ello, los objetivos generales y específicos del trabajo son:

1. Conocer la modalidad de gabinete de comunicación predominante.

1.1. Comprobar la implantación de gabinetes internos y externos en el Tercer Sector

1.2. Conocer si predomina el gabinete de prensa o los gabinetes han evolucionado hacia la gestión de la comunicación integral.

1.3. Conocer si la crisis ha influido en modelo de gabinete de comunicación y sus recursos.

2. Comprobar si la gestión de la comunicación en las ONGs objeto de estudio tiene una orientación estratégica o táctica.

2.1. Conocer si los Gabinetes disponen de un Plan de comunicación y relaciones públicas.

2.2. Comprobar si en la elaboración del Plan de comunicación y relaciones públicas se sigue algún modelo clásico de planificación estratégica de la comunicación y relaciones públicas, y concretamente el método RACE.

Con objeto de conseguir una muestra abarcable en relación a los objetivos propuestos, se ha centrado el estudio en la Comunidad Valenciana donde, como hemos apuntado, la crisis económica ha incidido de manera importante en la labor de las organizaciones del Tercer Sector.

Se ha seleccionado una muestra de ONG atendiendo a los siguientes criterios:

- ONG con sede en la Comunidad Valenciana y que desarrollen una labor representativa en la provincia de Valencia, por ser la capital donde se encuentran las sedes principales de las entidades del Tercer Sector que operan en dicha comunidad, siendo la única provincia en la que algunas ONG cuentan con departamento de comunicación.

- ONG que dirijan su trabajo hacia colectivos vulnerables y en riesgo de exclusión durante la crisis económica que atraviesa España, y con un número elevado de beneficiarios. Tras el análisis de Informes y documentos sobre este tipo de colectivos (FOESSA, 2008; Informe de Cruz Roja sobre Vulnerabilidad Social en la CV, 2012; etc.), todos coinciden 
en apuntar como grupos destacados: personas sin hogar y/o que reciben ayuda de emergencia y de alimentos; mujeres y hogares monoparentales; personas con discapacidad física o mental; inmigrantes, refugiados y minorías raciales; desempleados en general y otros colectivos vulnerables (personas en contacto con el sistema penitenciario, colectivos con problemas de drogodependencias, mayores...).

- $\quad$ ONG que tengan diferentes configuraciones jurídicas (asociaciones, federaciones, sociedad de utilidad pública...), distinta implantación (internacional, nacional, local) y diferencias en sus sistemas de funcionamiento, con objeto de que la muestra pueda ilustrar las diferentes realidades de un sector muy fragmentado y atomizado.

- ONG que dispongan de gabinete de comunicación con personal contratado.

Para identificar la muestra final de ONG en función de dichos criterios, se realizó una entrevista telefónica con las Plataformas de la Comunidad Valenciana que aglutinan a las principales entidades que trabajan mayoritariamente con dichos colectivos. Se entrevistó a los Coordinadores, Directores o Secretarios técnicos de las siguientes plataformas: Coordinadora de ONGD de la CV (CVONGD); Plataforma de Entidades de Voluntariado de la CV (PVSCV); Xarxa de las Xarxas de Acción Social y Cooperación; Comité Español de representantes de personas con discapacidad (CERMI); Valencia Acoge (Taula de Entidades de Solidaridad con los inmigrantes) y Agencia Valenciana de Cooperación Social (VLcsocial). En base a los criterios referidos anteriormente, designaron las ONG más representativas en Valencia que trabajan con los colectivos señalados y que disponen de gabinete de comunicación: Cruz Roja, Cáritas, Casa Caridad, CEARPV (Comisión Española de Ayuda al Refugiado en el País Valencià), Fundación COCEMFECV (Confederación de personas con discapacidad física y orgánica de la Comunidad Valenciana) y Asociación FEAPSCV (Federación de Asociaciones a favor de las personas con discapacidad intelectual en la Comunidad Valenciana).

Una vez seleccionadas las entidades objeto de estudio, se determinaron las personas que serían los interlocutores sobre quienes aplicar la metodología y técnicas de investigación elegidas, siendo los responsables de la gestión de la comunicación en dichas entidades (directores de departamentos internos, con la salvedad de Casa de la Caridad cuya gestión se realiza desde la Agencia de comunicación "Grupo R Comunicación").

La metodología utilizada ha sido cualitativa: entrevista en profundidad (Ruíz, 2003; Berganza y Ruíz, 2005; Tójar, 2006; Vallés, 2010) dirigida a los responsables de los gabinetes de comunicación; y realizando también una clasificación de los resultados de las entrevistas en función de las acciones realizadas por las ONGs en cada una de las fases del Modelo RACE.

Para la realización de las entrevistas en profundidad se elaboró un guion siguiendo los criterios marcados por diferentes autores de referencia en la aplicación de esta técnica de investigación (Galindo, 1998; Ruíz, 2003; Berganza y Ruíz, 2005; Vallés, 
2010), que se remitió por mail a los entrevistados. Al tratarse de una entrevista abierta, se ha contemplado la inclusión de otras cuestiones que surgieran a lo largo de la misma. Las entrevistas tuvieron lugar entre septiembre y octubre de 2013 en las sedes de las ONG, salvo en el caso de CEARPV que se realizó por skype ya que el responsable de comunicación se encontraba en Nicaragua durante 2013.

El guion estaba estructurado en tres áreas: sobre el gabinete (trayectoria, modalidad, ubicación e interacción en el organigrama, recursos humanos y DIRCOM...); estructura (áreas, funciones, medios y herramientas...); y Plan de comunicación (investigación, planificación, acción/ejecución y evaluación, objetivos y públicos...).

Por otra parte, se ha definido una categorización ad hoc aplicada al análisis de las respuestas de las entrevistas, y en particular las referidas a la metodología y procesos seguidos en la elaboración de los planes de comunicación de las ONG, para inferir el uso o no de modelos de planificación estratégica y, en particular, del Método RACE.

Las categorías elaboradas para comprobar la aplicación del Método RACE en base al análisis de las respuestas obtenidas de las entrevistas fueron las siguientes:

- Investigación

a) Investigación antes de plantear los planes a largo plazo (cada 3-5 años) con auditorias de imagen y sociales, o la aplicación de varias técnicas de investigación incluyendo análisis de percepciones de los públicos, realidad social, competencia... con fase final de diagnóstico (DAFO)

b) Investigación antes de plantear los planes a largo plazo (cada 3-5 años) con metodología más sencilla y focalizada en percepciones de públicos y fase de diagnóstico (DAFO)

c) Investigación anual u orientada a campañas concretas

d) Otros tipos de investigación orientada a la planificación estratégica

e) No realizan investigación orientada a la planificación estratégica

- Acción / Planificación

a) Planificación a largo, medio (anual) y corto plazo con definición de objetivos, públicos, estrategia de comunicación y planificación táctica

b) Planificación a largo y medio plazo (anual), con definición de objetivos, públicos, estrategia de comunicación y planificación táctica

c) Planificación anual (medio plazo) con objetivos y públicos, y estrategia y planificación táctica

d) Planificación a corto plazo (campañas y acciones) con definición de objetivos, públicos, estrategia de comunicación y planificación táctica

e) No realizan planificación 
- Comunicación

a) Planes de acción anuales y calendarios anuales de ejecución y revisiones intermedias (trimestrales, mensuales...)

b) Planes de acción anuales y calendarios anuales de ejecución

c) Planes temáticos o por tácticas con calendario de ejecución por tácticas

d) No existen planes con calendarios de ejecución identificados

- Evaluación

a) Evaluación sumativa de la consecución de objetivos y en los diferentes públicos (llevada a cabo al final del Plan) y formativa (llevada a cabo mientras el Plan está en marcha)

b) Solo evaluación sumativa

c) Solo evaluación formativa

d) No realizan una evaluación de sus planes, campañas o acciones

\section{Resultados}

\subsection{Modalidad de gabinetes según ubicación, fecha de inicio, denominación y trayectoria}

Se constata que cinco de los seis gabinetes analizados son internos y sólo uno externo (Casa Caridad), aunque Cruz Roja contrata además los servicios de la Agencia Kantar Media a para realizar el clipping de medios (a nivel regional).

Como se aprecia en el Gráfico 1, la trayectoria de la mayoría de ellos es corta, siendo los dos más antiguos los de Cruz Roja y Cáritas (en los años 90). Los otros cuatro surgen a lo largo de la década del 2000: FEAPSCV hace 10 años; COCEMFECV y CEARPV hace 9 años y Casa Caridad hace 6 años (aunque desde dos años antes ya gestionaban algunos temas relacionados con la comunicación a través de los servicios de la Agencia Grupo R Comunicación).

Gráfico 1. Fecha de inicio de los gabinetes.

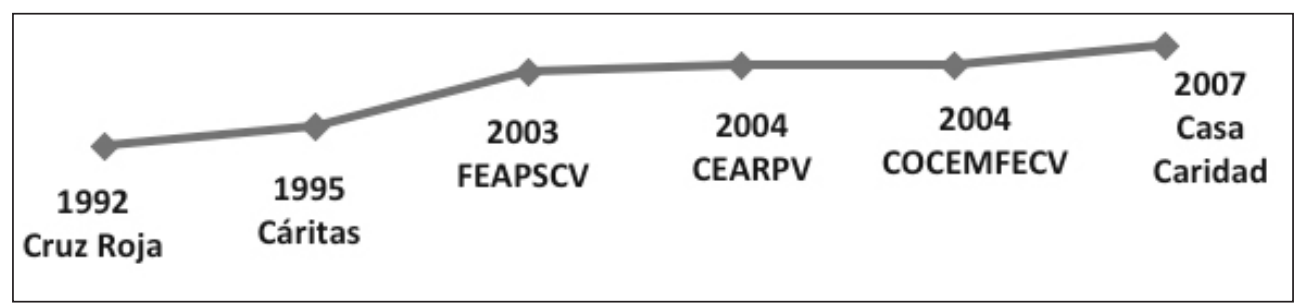

Respecto a la denominación adoptada por cada uno y su trayectoria o evolución en cuanto al modo de gestionar la comunicación (Tabla 1): 
- Denominación: cada gabinete adopta un nombre distinto, aunque en cuatro de ellos observamos que está presente el término "comunicación": en dos casos su denominación es "departamento de comunicación" (Cruz Roja y FEAPSCV); en uno "servicio de comunicación" (COCEMFECV) y en otro "departamento de marketing y comunicación” (Casa Caridad). Además, en dos ONG la comunicación está integrada en áreas más amplias con otras denominaciones: "departamento de sensibilización" (Cáritas) e "incidencia, participación social y voluntariado" (CEARPV).

- Trayectoria como gabinetes: cuatro de ellos desde el principio se pusieron en marcha para gestionar la comunicación de manera integral (Cruz Roja, Cáritas, Casa Caridad y CEARPV) y dos comenzaron como gabinetes de prensa, ampliando poco a poco sus funciones (COCEMFECV y FEAPS). En este sentido, en todos los casos la evolución ha sido hacia un modelo de Gabinete de comunicación integral.

Tabla 1. Denominación y trayectoria de los gabinetes.

\begin{tabular}{|l|l|l|}
\hline ONG & Denominación & Cómo surgen y evolucionan \\
\hline Cruz Roja & $\begin{array}{l}\text { Departamento de } \\
\text { Comunicación }\end{array}$ & $\begin{array}{l}\text { Se crea para coordinar toda la } \\
\text { comunicación, aunque inicialmente más } \\
\text { orientado a la comunicación interna. }\end{array}$ \\
\hline Cáritas & $\begin{array}{l}\text { Departamento de } \\
\text { Sensibilización }\end{array}$ & $\begin{array}{l}\text { El área de comunicación (con todas } \\
\text { las funciones propias del gabinete) } \\
\text { está integrada desde el principio en un } \\
\text { departamento más amplio. }\end{array}$ \\
\hline Casa Caridad & $\begin{array}{l}\text { Departamento } \\
\text { de Marketing y } \\
\text { Comunicación }\end{array}$ & $\begin{array}{l}\text { Desde el principio surge integrando ambas } \\
\text { funciones. }\end{array}$ \\
\hline CEARPV & Incidencia, Participación & $\begin{array}{l}\text { Esta área más amplia integra desde } \\
\text { el comienzo las funciones propias del } \\
\text { departamento de comunicación. }\end{array}$ \\
\hline COCEMFECV & $\begin{array}{l}\text { Servicio de } \\
\text { Comunicación }\end{array}$ & $\begin{array}{l}\text { Comienza con la finalidad de enviar notas } \\
\text { de prensa (gabinete de prensa) y elaborar } \\
\text { una revista corporativa. Poco a poco va } \\
\text { ampliando funciones hasta la actualidad. }\end{array}$ \\
\hline FEAPSCV & $\begin{array}{l}\text { Departamento de } \\
\text { Comunicación }\end{array}$ & $\begin{array}{l}\text { Surge con este nombre, pero centrado en } \\
\text { las relaciones con los medios (gabinete de } \\
\text { prensa) y elaboración de folletos. Poco a } \\
\text { poco va ampliando funciones hasta hoy. }\end{array}$ \\
\hline
\end{tabular}




\subsection{Modelo de gabinete en función de los recursos humanos, modalidad laboral y su perfil profesional}

En cuanto al número de personas que trabajan en cada gabinete, su modalidad laboral -contratadas, voluntarias, en prácticas y personal externo-, así como la titulación de las personas contratadas:

La ONG que dispone de más recursos humanos es Cruz Roja con 7 personas: 1 contratada, 5 voluntarias (1 para redes sociales, 2 en la Unidad de comunicación de emergencias en Valencia-UCE-, 1 fotógrafo, 1 persona para ayudar en campañas), y 1 en prácticas. Le sigue Casa Caridad con 5 personas: 2 realizan los servicios contratados a la Agencia Grupo R comunicación (gabinete de prensa, redes sociales, memoria e Informe de pobreza anual, parte de la web y apoyo en eventos), 1 contratada en la Agencia Dimarco (publicidad y otros) y 2 voluntarios (uno pertenece a la Junta directiva y coordina todas las acciones de comunicación, y 1 que colabora en las relaciones externas). La siguiente ONG es Cáritas con 2 personas en 2012: 1 contratada y 1 voluntaria que realiza el clipping de medios (además, desde el 2013 otra persona voluntaria coordina las redes sociales). Las ONG CEARPV, COCEMFECV y FEAPS cuentan sólo con 1 persona contratada en cada entidad que se ocupa de la totalidad de funciones del gabinete.

Respecto al perfil profesional de las personas contratadas, en cinco ONG proceden del ámbito de las Ciencias de la Información (Licenciado en Periodismo) y únicamente en el gabinete de CEARPV encontramos un perfil profesional diverso (Licenciado en Ingeniería Informática).

\subsection{Consideración del gabinete de comunicación por la dirección e interacción con otros departamentos}

Los responsables de comunicación entrevistados han señalado que la gestión de la comunicación en sus entidades es considerada un factor estratégico al servicio de los fines de la organización, debido a una creciente concienciación por parte de sus Juntas de gobierno. Prueba de ello es que todos los gabinetes se han mantenido en momentos de crisis económica con el mismo número de personas, ampliando incluso con algún voluntario.

Hemos querido conocer además la ubicación del departamento en el organigrama de las ONG y el papel desempeñado por la persona que asume su dirección en el conjunto de funciones de la entidad con el fin de valorar el carácter estratégico del puesto. Como se ha señalado al tratar sobre la denominación de los gabinetes, cuatro de ellos (Cruz Roja, Casa Caridad, COCEMFECV y FEAPSCV) son independientes y realizan las tareas propias de un departamento de comunicación. Este departamento tiene una ubicación en el organigrama de las ONG al mismo nivel que el resto de departamentos dentro de las ONG. En el caso de Cáritas, el área de comunicación está integrada en un departamento más amplio (sensibilización), aunque con un responsable que se dedica en exclusiva a la misma. En CEARPV en cambio la comunicación se integra también en otra área (Incidencia, Participación y Voluntariado), con la diferencia de que su responsable se ocupa de todas las funciones propias de esta área, incluida la comunicación. 
En tres ONG (Cruz Roja, COCEMFE y FEAPSCV) el responsable de comunicación es considerado DIRCOM, aunque sólo en dos de ellas -Cruz Roja y FEAPSCVse encuentra integrado en el managment de la organización (realizando funciones de asesoramiento a dirección). En Casa Caridad (Agencia externa) la coordinación del departamento la realiza un miembro voluntario del Comité directivo de la entidad, y en Cáritas y CEARPV quien dirige estas funciones es considerado "responsable de comunicación”, pues está integrado en un área más amplia.

Todos los responsables de los gabinetes manifiestan que existe una estrecha colaboración entre dicha área y el resto de departamentos de la organización, en especial con el de voluntariado y captación de fondos. La colaboración es especialmente estrecha con las áreas de proyectos, pues en casi todos existe un apartado destinado a comunicación (a veces denominado Plan de comunicación), que suele encargarse de elaborar y desarrollar el propio gabinete de comunicación.

\subsection{Modelo de gabinete en función de su estructura, áreas de gestión / funciones}

En la Tabla 2 desarrollamos las funciones y tácticas comunes a todos los gabinetes, de lo que se desprende que todos ellos gestionan la comunicación de manera integral, con algunas diferencias:

Tabla 2. Funciones desarrolladas por los gabinetes.

\begin{tabular}{|l|l|}
\hline Funciones comunes en todos los gabinetes & Diferencias en algunas funciones \\
\hline - Comunicación interna & - Publicidad: sólo Cruz Roja y Casa \\
- Manual de identidad e imagen corporativa: & Caridad invierten en acciones. Cáritas \\
orientaciones a nivel nacional que adaptan & difunde algún spot gratuito en Radio o \\
en Valencia & TV (a nivel nacional) \\
- Gabinete de prensa & - Captación de fondos y RSC: áreas \\
- Portavoces y formación: para los miembros & independientes del Gabinete de \\
de la dirección, técnicos, voluntarios, etc. & comunicación, con diferentes niveles \\
que intervienen en los medios & de colaboración con él mismo \\
- Relaciones con la comunidad: todos & - Unidad de comunicación de \\
implicados en la organización de eventos & emergencias (UCE): desarrollada por \\
(Cáritas y CoCEMFECV sólo en cuanto a & Cruz Roja \\
relaciones con los medios) & - Redes Sociales: en 2012 en \\
- Publicaciones: salvo CEARPV, todas & desarrollo incipiente en todas las \\
participan habitualmente en la redacción de & ONGs (sólo Casa Caridad tiene \\
Memoria y/o Informe anual y Boletín periódico & estrategia) \\
- Comunicación de crisis: poco desarrollada & \\
en todas; siguen lo señalado a nivel nacional & \\
- Página web: ninguna es 2.0 & \\
\hline
\end{tabular}


En cuanto a la comunicación digital como una función de los gabinetes y la situación en que se encuentran respecto a la utilización de las redes sociales: su incorporación al trabajo de los Gabinetes comienza en el año 2009 con Facebook (Cruz Roja y CEARPV), primera red utilizada por cuatro de las ONG (Cáritas no la incorpora hasta el 2013). Twitter es la red de más reciente incorporación (a partir de 2011) y en el año objeto de nuestro estudio (2012) Cáritas y FEAPSCV aún no la habían incorporado (lo hacen en el 2013); en el caso de CEARPV todavía no se utilizaba en dicha fecha.

Sobre el uso estratégico de estas redes, el gabinete de Casa Caridad fue el primero en desarrollar una estrategia para ambas (en 2011), que Cruz Roja y Cáritas incorporan en 2013. Las demás ONG se están iniciando con estos canales sin tener una estrategia claramente desarrollada en el momento de realizar la entrevista. Y respecto a la responsabilidad de gestionarlas, en cuatro ONG son los propios responsables del gabinete de comunicación (interno o externo) y en dos de ellas lo hace un voluntario (Cruz Roja y Cáritas).

\subsection{Plan de comunicación y relaciones públicas e influencia de la crisis en los objetivos de comunicación}

Todas las ONG analizadas cuentan con un Plan estratégico de la entidad que se elabora en la sede nacional (salvo Casa Caridad, ONG local) y que se traslada a Valencia con las adaptaciones oportunas. En dichos planes destaca el papel relevante de la comunicación -especialmente en los elaborados los últimos trienios-, concretándose en un Plan de comunicación nacional, que se adapta a nivel regional.

Como se aprecia en el Gráfico 2, aunque todos los gabinetes inician su trayectoria con un planteamiento más bien táctico en su gestión de la comunicación, empiezan a incorporar progresivamente otro más estratégico mediante la elaboración de planes a partir del año 2000, siendo pionero el de Cruz Roja (2001) y el más reciente el de COCEMFECV (2012).

Gráfico 2: Fecha creación del gabinete y del primer Plan de comunicación.

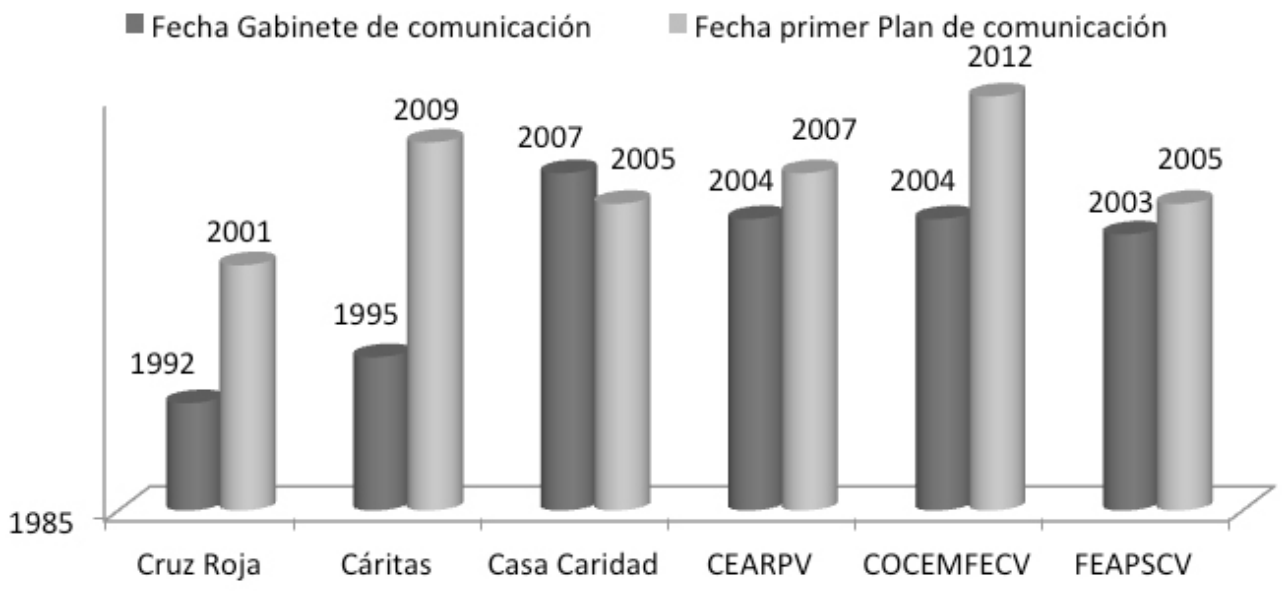


Todos constatan que diseñan sus propios Planes de comunicación, y cinco de ellos toman como base los Planes de comunicación nacionales, haciendo una adaptación de objetivos, públicos y herramientas a las circunstancias y necesidades locales (salvo Casa Caridad). En todos ellos, la dirección de la entidad conoce y aprueba dicho plan, que ha sido elaborado por el responsable del gabinete, contando con la opinión y necesidades de todos los departamentos. Una vez aprobado, se traslada a cada departamento de la ONG para aquello que le compete, siendo Cáritas -desde 2013la única que ha comenzado a presentar de manera oficial el Plan de comunicación, reuniendo a todos los departamentos de su organización.

Aunque las seis ONG objeto de estudio realizan una planificación detallando públicos y objetivos en sintonía con los de sus entidades, algunas de ellas han señalado diversas particularidades motivadas por la situación de crisis económica. Para Cruz Roja, Cáritas y Casa Caridad uno de sus objetivos ha sido aprovechar el aumento de requerimientos por parte de los medios, y en el caso de Cruz Roja la realización de la campaña "Ahora + que nunca". Como resultado, las tres entidades han experimentado un aumento en su publicity, así como un incremento del número de socios, donantes y voluntarios.

En los casos de CEARPV, COCEMFECV y FEAPS, el incremento de informaciones enviadas a los medios ha estado relacionado sobre todo con la denuncia por los recortes de ayudas económicas por parte las instituciones públicas, con el fin de visualizar la situación de las entidades y sus beneficiarios durante la crisis; también han realizado diversas acciones de comunicación en red con otras ONG para promover la movilización social ciudadana frente a las instituciones públicas. Pero las tres han reflejado que dichas acciones no se ha traducido en un incremento relevante de la publicity ni en una mejora de los resultados de sus entidades.

\subsection{Seguimiento o aplicación del Método RACE en la elaboración de los planes de comunicación}

A continuación exponemos el análisis de los resultados de las entrevistas en la parte relativa a las fases seguidas en la elaboración del Plan de comunicación aplicando las categorías descritas en el capítulo metodológico:

- Por lo que se refiere a la fase de INVESTIGACIÓN, se constata que únicamente Cruz Roja y Cáritas realizan cierta investigación antes de plantear los planes a largo plazo (cada 3-5 años), con metodología más sencilla y focalizada en percepciones de públicos y fase de diagnóstico (DAFO). En el caso de Casa Caridad, realiza cierta investigación anual orientada a campañas concretas - por ejemplo el estudio del origen de los socios para plantear las campañas de captación de fondos-; y en los gabinetes de CEARPV, COCEMFECV y FEAPSCV no realizan ninguna investigación orientada a la planificación estratégica.

- En cuanto a la ACCIÓN/PLANIFICACIÓN, tanto Cruz Roja, como Cáritas y Casa Caridad realizan una planificación a largo, medio y corto plazo con definición de objetivos, públicos, estrategia de comunicación 
y planificación táctica. En los casos de CEARPV, COCEMFECV y FEAPSCV realizan una planificación anual con objetivos y públicos, y estrategia y planificación táctica.

- En la fase COMUNICACIÓN, cuatro de las ONG realizan planes de acción anuales, calendarios anuales de ejecución y revisiones intermedias (trimestrales, mensuales...): Cruz Roja, Cáritas, Casa Caridad y FEAPS; en el caso de COCEMFECV, realizan planes de acción anuales y calendarios anuales de ejecución, y en CEARPV no han reflejado que existan planes con calendarios de ejecución identificados.

- Por lo que se refiere a la EVALUACIÓN, en todos los casos se trata fundamentalmente de una evaluación focalizada en medir la exposición e impacto en los medios con técnicas cuantitativas y/o cualitativas con uno de sus públicos (los medios de comunicación). Todos los gabinetes realizan evaluación cuantitativa (siendo la más completa la de Cruz Roja y Casa Caridad); dos de ellos cualitativa parcial (Cruz Roja y Casa Caridad); y únicamente Cruz Roja realiza evaluación económica. COCEMFECV señala que también efectúa una medición de su comunicación digital contabilizando el número de suscriptores a su revista y boletín, así como las visitas web y los fans en redes sociales. CEARPV realiza en parte evaluación sumativa centrada en actitudes y comportamientos, midiendo el nivel de adhesión y movilización social conseguido por la entidad, y los cambios producidos en las políticas públicas de refugio e inmigración como consecuencia de su trabajo.

En definitiva, los resultados presentados reflejan que aunque todas las ONG realizan sus Planes de comunicación centrándose principalmente en la acción/planificación y comunicación (calendario de ejecución), Cruz Roja, Cáritas y Casa de la Caridad tienen un planteamiento más estratégico trabajando en el largo, medio y corto plazo con una programación de su fase táctica. Además, algunas ONG realizan un mayor esfuerzo en materia de investigación (Cruz Roja y Cáritas), destacando el caso de CEARPV donde encontramos en parte evaluación sumativa que va más allá de la medición de la exposición e impacto en medios, evaluando el nivel de adhesión y movilización social y los cambios producidos en las políticas públicas como consecuencia de su trabajo.

\section{Conclusiones}

1. Todas las ONG cuentan con gabinetes de comunicación, de reciente creación, y en su mayoría internos salvo uno externo (Casa Caridad).

2. Predominan los departamentos que incluyen en su denominación el término "comunicación" y que comienzan desde el principio a gestionar la comunicación de manera integral, dirigiéndose a más públicos y gestio- 
nando otras áreas y funciones además de las relaciones con los medios. Todos estructuran la gestión de su comunicación en torno a la comunicación interna y externa, asumiendo un número elevado de funciones comunes: comunicación interna; relaciones con los medios; la función de portavoz/es y su formación; relaciones con la comunidad y organización de eventos; publicaciones y manual de imagen corporativa. Solo en dos casos (COCEMFECV y FEAPSCV) el origen ha sido un gabinete de prensa.

3. La mayoría de los gabinetes cuentan con recursos limitados, constatando la escasez de personal contratado y su combinación con personal voluntario ( sin conocimiento estratégico de la comunicación y con una función más técnica en varias de las $\mathrm{ONG}$ ), lo que resulta indicativo de una falta de profesionalización de la comunicación. Además, observamos una falta de profesionales con perfil de relaciones públicas, predominando el de licenciado en periodismo, más propio del Gabinete de prensa.

4. Los gabinetes de comunicación se han mantenido durante la crisis, lo que indica su consideración como área clave dentro de estas organizaciones. No obstante, sólo en tres ONG (Cruz Roja, COCEMFE y FEAPSCV) encontramos la figura del DIRCOM, aunque únicamente en Cruz Roja y FEAPASCV está integrado en el managment de la organización, por lo que su función de asesoramiento y participación en la toma de decisiones es todavía limitada.

5. Por otra parte, si atendemos a la orientación estratégica o táctica de la comunicación, hemos encontrado dos modelos diferenciados en la gestión de la comunicación: por un lado, en Cruz Roja, Cáritas y Casa Caridad existe un planteamiento estratégico más en línea con el modelo RACE, adaptando sus objetivos de comunicación en los momentos de crisis económica (rentabilizando, por ejemplo, en términos de publicity el mayor interés de los medios por sus actividades); en los casos de CEARPCV, COCEMFECV y FEAPSCV su planteamiento es más táctico, lo que ha podido influir en no rentabilizar adecuadamente, su presencia en medios durante la crisis. Así, aunque todos los gabinetes objeto de estudio inician su trayectoria con un planteamiento táctico en la gestión de la comunicación, a partir de 2000 y hasta 2012 se va incorporando progresivamente un planteamiento estratégico en sus Planes de comunicación, si bien solo en las entidades con más trayectoria (Cruz Roja y Cáritas, seguidas de Casa Caridad) el Plan incluye verdaderamente objetivos a largo, medio y corto plazo. En esto puede influir también la presencia de voluntarios que en su mayoría no tienen conocimientos estratégicos de comunicación.

6. Se concluye, además, que Cruz Roja, Cáritas y Casa Caridad son las ONG con mayor nivel de seguimiento del modelo RACE; si bien la mayoría de los gabinetes elaboran sus Planes de comunicación centrándose 
principalmente en la Acción/Planificación y la Comunicación (calendario de ejecución), por lo que la investigación y la evaluación siguen siendo asignaturas pendientes, con algunas excepciones señaladas.

Por lo tanto, y en base a las conclusiones referidas, la hipótesis principal queda validada sólo para Cruz Roja, Cáritas y Casa Caridad. En los casos de CEARPCV, COCEMFECV y FEAPSCV, aunque también han evolucionado hacia un Departamento de comunicación integral, no encontramos una orientación estratégica en la gestión de su comunicación en línea con el modelo RACE.

\section{Referencias bibliográficas}

ALMANSA, Ana (2011). Del Gabinete de prensa al Gabinete de comunicación: la dirección de la comunicación en la actualidad. Zamora: Comunicación Social.

ARAGONÉS, P. (1998). Empresa y medios de comunicación. Barcelona: Ediciones Gestión 2000.

ÁLVAREZ, M ${ }^{\mathrm{a}}$ Isabel (2012). La profesionalización de la Comunicación para la Solidaridad: diagnóstico y propuestas para el sector en España. En: Cuadernos de Información y Comunicación, $\mathrm{n}^{\circ} 17$, pp. 239-253.

BALAS, Montserrat (2010). La gestión de la comunicación en el Tercer Sector. (Tesis de doctorado, Universidad Jaime I de Castellón). Recuperado de: http://hdl.handle.net/10234/14936

BALAS, Montserrat (2011). La gestión de la comunicación en el Tercer Sector. Madrid: ESIC.

BEL, José Ignacio (2004). Comunicar para crear valor. Pamplona: EUNSA.

BERGANZA, Ma Rosa; RUÍZ, José Ignacio (2005). Investigar en comunicación. Madrid: McGraw-Hill. Interamericana de España.

BERNABÉ, Javier (2001). Las áreas de comunicación de las ONGD y el trabajo periodístico. En: Revista española de Cooperación y Desarrollo, $\mathrm{n}^{\circ}$ 7, pp. 127-141.

BERNAYS, Edward (1996). Relaciones públicas. Buenos Aires: Tronquel.

BLACK, S. (1994). Las Relaciones Públicas. Un factor clave de gestión. Barcelona: Hispanoeuropea.

CANEL, Mª José. (2007). Comunicación en las instituciones públicas. Madrid: Tecnos. CASTILLO, Antonio (2009). Relaciones Públicas. Teoría e historia. Barcelona: UOC.

CASTILLO, Antonio y ALVÁREZ, Alejandro (2015). Evaluación en comunicación estratégica. Madrid: McGraw-Hill.

CERVERA, Angel Luis (2008). Comunicación total. Madrid: ESIC.

CORNELISSEN, Joep \& HARRIS, Phil (2001). The Corporate Identity Metaphor: Perspectives, Problems and Prospects. En: Journal of Marketing Management, $\mathrm{n}^{\circ}$ 17(1/2), pp. 49-71. 
COSTA, Joan (2009). DirCom. Estratega de la complejidad. València: Publicacions Universitat de València.

CUTLIP, S. y CENTER, A. H. (1952). Effective Public Relations. Englewood Cliffs: Prentice Hall.

DE LA RIVA, Fernando (2002) (Coord.). El Tercer Sector visto desde dentro. La renovación de las ONGs y los retos de la exclusión social. Barcelona: Fundación ESPLAI.

DEL POZO, M ${ }^{\mathrm{a}}$ Luisa (2000). Gestión de la comunicación interna en las organizaciones. Pamplona: EUNSA.

DURÁN, Patricia; FERNÁNDEZ, María Belén (2010). La comunicación en las organizaciones del tercer sector. En: Revista Latina de Comunicación Social, $\mathrm{n}^{\mathrm{o}}$ 65, pp. 595-603.

ERRO, Javier; VENTURA, Javier. (2002). El trabajo de comunicación de las ONGD en el País Vasco. Bilbao: Hegoa.

FERNÁNDEZ, Ma Jesús (2005). Las Relaciones Públicas como gestión de lacomunicación en los movimientos sociales. Análisis de la estrategia comunicativa de las ONGs en España. (Tesis de doctorado, Universidad de Málaga).

GALINDO, Jesús (Coord.). (1998). Técnicas de investigación en sociedad, cultura y comunicación. Mexico: Pearson Educación (Addison Wesley).

GARCÍA, Berta (2005). Los altavoces de la actualidad: Radiografía de los gabinetes de comunicación. La Coruña: Netbiblo.

GARCÍA, Berta (2006). Aproximación teórica a la comunicación en el tercer sector. La necesaria reclasificación de la comunicación organizacional. En: TELOS, Cuadernos de Comunicación e Innovación, $\mathrm{n}^{\circ}$ 69, pp. 51-59.

GONZÁLEZ, Hildegart (2005). Estrategias de comunicación e impacto mediático de las ONGS (Tesis de doctorado, Universidad de Navarra).

GONZÁLEZ, Hildegart (2006). Estrategias de comunicación en las ONG de desarrollo: Departamentos, funciones e impacto en los medios. Madrid: CIDEAL.

GONZÁLEZ, Ma Isabel (2010). Comunicación para la Solidaridad: las ONG y el papel de la comunicación social y periodística en la sensibilización de la opinión pública y el desarrollo. (Tesis de doctorado, Universidad Complutense de Madrid). Recuperado de: http://eprints.ucm.es/11633/

GUTIÉRREZ-DOMÊNECH, María (2013). Radiografía poscrisis de la pobreza en España,Informe Mensual.La Caixa, n 366,pp.52-55. Recuperado en: http://www. lacaixaresearch.com/documents/10180/55077/IM+366+Marzo+2013+CAST. pdf/25d63542-877f-4533-aa8d-f044defdd6ca

GUTIÉRREZ-GARCÍA, Elena; LAPORTE, Ma Teresa (Eds.) (2013). Tendencias emergentes en la comunicación de instituciones. Madrid: UOCpress.

HAINSWORTH, B. E. y WILSON, L. J. (1992). Strategic Program Planning. Public Relations Review, 18 (1), 11.

HERNÁNDEZ, Ana (2002). Planificar la comunicación. En: Revista Latina de comunicación Social, no 48, vol. 5. Recuperado en: http://www.ull.es/publicaciones/latina/2002/latina48marzo/4811gonzalez.htm

HERRANZ DE LA CASA, José María (2009). Comunicación y transparencia en las organizaciones sociales. En: Revista de comunicación y Nuevas tecnologías ICONO 14, n 13 , pp. 172-194. 
HERRANZ DE LA CASA, José María (2010). La comunicación y la transparencia en las organizaciones no lucrativas. (Tesis de doctorado, Universidad Complutense de Madrid). Recuperado en: http://eprints.ucm.es/11539/

KROHLING KUNSCH, Margarida (2005). Planejamento de Relacoes Publicas. En CASTILlO, A. (Coord.). Comunicación Organizacional, Teorías y Estudios, pp. 75-95. Málaga: Aynadamar.

L'ETANG, Jacquie (2009). Relaciones públicas. Conceptos, práctica y crítica. Barcelona: UOC.

MARTÍN, Fernando (2006). Comunicación Empresarial e Institucional (4 $\left.{ }^{\mathrm{a}} \mathrm{Ed}.\right)$. Madrid: Universitas.

MARTÍNEZ, José Luis (1998). La Imagen de las ONG de desarrollo. Madrid: IEPALA.

MATILLA, Kathy (2007). Aportaciones para un modelo global de Planificación Estratégica en Relaciones Públicas y Comunicación integral. Análisis de un caso. (Tesis de doctorado, Universidad Ramón Lull, Barcelona). Recuperado de: http:// hdl.handle.net/10803/9222

MATILLA, Kathy (2008). Los modelos de planificación estratégica en la teoría de las Relaciones Públicas. Barcelona: UOC.

MATILLA, Kathy (2009). Aportaciones para un nuevo Modelo de Planificación Estratégica en Relaciones Públicas. Trabajo presentado en FISEC-EstrategiasFacultad de Ciencias Sociales de la Universidad Nacional de Lomas de Zamora, 12(2), Año V, 17 32. Recuperado de: http://www.cienciared.com.ar/ra/usr/9/689/ fisec_estrategias_n12_m2pp17_32.pdf

MATILLA, Kathy (2011). La función estratégica del Dircom en España en el 2010. En: Revista Internacional de Relaciones Públicas, vol I (2), 11-23.

MOLINA, Juan Pedro (2007). La profesionalización de los directores de comunicación en las ONG. Madrid: Edipo.

PAGÁN, Juan Antonio (2009). El futuro de los gabinetes de comunicación. En: $R e$ vista de Comunicación, $\mathrm{n}^{\circ} 12$, pp. 40-43.

PARICIO, Pilar y REGADERA, Elisa (2014). Gestión de la comunicación y las Relaciones Públicas en Gabinetes de ONGs en Valencia. Las Relaciones con los Medios en los Gabinetes de Cruz Roja, Cáritas, Manos Unidas, Fontilles, Casa Caridad y Casa Cuna Santa Isabel. En: Sphera Pública, $\mathrm{n}^{\circ}$ especial, pp. 21-43.

PAVLIK, John (1999). La investigación en las relaciones públicas. Barcelona: Gestión 2000.

PÉREZ, Rafael Alberto (2008). Estrategias de comunicación. Barcelona: Ariel.

PÉREZ VARELA, Jesús (1996). “Clausura del seminario”. En MACIÁ MERCADÉ, Juan. Los Gabinetes de prensa, alternativa profesional. Madrid: S.A. Ciencia 3.

RAMÍREZ, Txema (1995). Gabinetes de comunicación. Barcelona: Bosch.

REGADERA, Elisa y PARICIO, Pilar (2014). Análisis de la gestión y los Programas de relaciones con los medios en Gabinetes de Comunicación de ONG en Valencia (2011). En: Derecom, no 17, pp. 56-72.

ROMERO, Alfredo (2008). Organizaciones no gubernamentales, medios de comunicación y nuevas tecnologías. La visión de un jurista. En: Aposta, n 37, pp. 1-19.

RUíZ, José Ignacio (2003). Metodología de la investigación cualitativa. Bilbao: Universidad de Deusto. 
SANCHÉZ MORALES, Ma del Rosario (2013). Nueva pobreza y personas "sin hogar" en España. En Temas para el debate, n 218-219, pp. 51-53.

SRIRAMESH, Krishnamurthy y VERCI, Dejan (2012). Relaciones Públicas globales. Barcelona: UOC.

SORIA, Ma del Mar (2010). Estructura actual de los Departamentos de Comunicación de las ONGs españolas: de la persuasión a la gestión de las relaciones públicas. Trabajo presentado en el II Congreso Internacional Latina de Comunicación Social, Universidad de La Laguna (Actas), septiembre. Recuperado: http:// www.revistalatinacs.org/10SLCS/actas_2010/056_Soria.pdf

TÓJAR, Juan Carlos (2006). Investigación cualitativa. Comprender y actuar. Madrid: La Muralla.

VALLES, Miguel (2010). Entrevistas cualitativas. Madrid: Centro de Investigaciones Sociológicas.

VERNIS, Alfred; IGLESIAS, María; SANZ, Beatriz; URGELL, Jaume; VIDAL, Pau (1998). La gestión de las organizaciones no lucrativas. Bilbao: Deusto.

VILLAFAÑE, Justo (2008). La gestión profesional de la imagen corporativa. Madrid: Ediciones Pirámide.

WILCOX, Dennis; CAMERON, Glen; XIFRA, Jordi (2012). Relaciones públicas. Estrategias y Tácticas $\left(10^{\mathrm{a}} \mathrm{Ed}\right)$. Madrid: Pearson Addison Wesley.

XIFRA, Jordi (2005). Planificación estratégica de las Relaciones Públicas. Barcelona: Paidós.

XIFRA, Jordi (2008). Modelos de las Relaciones Públicas Políticas: Análisis de la situación en Cataluña. En: Revista Latina de Comunicación social, $\mathrm{n}^{\mathrm{o}}$ 63, pp. 392-399. 